\title{
Examining variation across treatment clinics in cancer patients' psychological outcomes: results of a cross sectional survey
}

\author{
Mariko Carey ${ }^{1,2}$ (D) Robert Sanson-Fisher ${ }^{1,2} \cdot$ Tara Clinton-McHarg $^{1,2} \cdot$ Allison Boyes $^{1,2} \cdot$ Ian $^{1,2}$ Olver $^{3}$. \\ Christopher Oldmeadow $^{2} \cdot$ Christine Paul $^{1,2} \cdot$ Catherine D'Este $^{4} \cdot$ Frans Henskens $^{5}$
}

Received: 31 August 2017 / Accepted: 28 March 2018 / Published online: 4 April 2018

(C) The Author(s) 2018

\begin{abstract}
Purpose The majority of research on psychological outcomes for cancer patients has focussed on the role of individual characteristics, and disease and treatment factors. There has been very little exploration of the potential contribution of the treatment clinic to these outcomes. This study explored whether there is variation among clinics in cancer patients' psychological outcomes.

Methods Cancer outpatients were recruited from 22 medical oncology and haematology clinics in Australia. Participants completed a pen and paper survey including the Hospital Anxiety and Depression Scale (HADS), as well as sociodemographic, disease and treatment characteristics.

Results Of those eligible to participate, 4233 (82\%) consented and 2811 ( $81 \%$ of consenters) returned the completed survey. There was no statistically significant variation in HADS depression scores across clinics. Some difference in anxiety scores derived from the HADS questionnaire between clinics $(p=0.03)$ was found with the percentage of between-clinic variation estimated to be $1.11 \%$. However, once all demographic, disease and treatment predictors were adjusted for there was no statistical differences between clinics (percent of between-clinic variation $=0.53 \% ; p=0.1415$ ).

Conclusions Psychological outcomes were not found to vary between clinics. Other sources of variation including patient characteristics may over-ride between-clinic variability, if it exists.
\end{abstract}

Keywords Cancer · Oncology $\cdot$ Psychological $\cdot$ Anxiety $\cdot$ Depression $\cdot$ Quality of care

\section{Background}

There is an abundance of research indicating that people with cancer experience high rates of depression and anxiety [1-3]. Despite recognition of the importance of addressing adverse

Mariko Carey

Mariko.Carey@newcastle.edu.au

1 Priority Research Centre for Health Behaviour, School of Medicine and Public Health, The University of Newcastle, W4 HMRI Building, Callaghan, NSW 2308, Australia

2 Hunter Medical Research Institute, New Lambton, NSW 2305, Australia

3 Samson Institute for Health Research, University of South Australia, Adelaide, SA 5001, Australia

4 National Centre for Epidemiology and Population Health, Research School of Population Health, Australian National University, Canberra, ACT 2601, Australia

5 School of Electrical Engineering and Computer Science, The University of Newcastle, Callaghan, NSW 2308, Australia psychological outcomes, these conditions remain underdetected and under-treated in many oncology and haematology settings $[4,5]$. Consequently, there is a need to delineate the contributing factors that may have an effect on patient psychological outcomes.

\section{What factors may influence psychological outcomes for people with cancer?}

Non-modifiable individual characteristics such as age and gender [2] have been associated with psychological outcomes. Potentially modifiable individual factors such as coping strategies [6] and social support [7] have also been linked to poorer psychosocial outcomes. The association between disease and 
treatment characteristics has also been examined. Compared to other people with cancer, those undergoing current treatment [8], and people diagnosed with poor prognosis cancers such as lung, or cancers with complex treatment regimens such as haematological cancers tend to have poorer psychological outcomes $[2,9,10]$.

It is also possible that there are differences between clinics in patient psychosocial outcomes. Variation in patient outcomes has been observed across a number of other areas of health care. For example, the Dartmouth Atlas of Health Care has documented a fivefold regional variation in rate of leg amputations for patients with diabetes [11]. This may be due to regional variation in the management of diabetes across indicators such as blood glucose testing and control, blood cholesterol testing and control, smoking cessation and foot care to identify and treat ulcers [11]. Similarly, regional variation in unplanned readmissions following treatment for peripheral artery disease ranged from 1 in 3 to 1 in 10 [11]. After adjustment for casemix, analyses of national claims data in the US showed that variability in mortality following cancer resection was influenced by both hospital volume and surgeon volume [4].

Several other studies have provided evidence of variation in factors that potentially relate to psychological outcomes. Jacobsen and colleagues reported variation in indicators of psychological care across 11 medical outpatient clinics. The study relied on medical records data, so it was unclear to what extent the variation observed related to actual differences in care patterns or to variation in the quality of documentation. Variation in patients' psychological outcomes was not examined. There is some evidence of variation in related outcomes such as patient experience and pain across cancer clinics. A Korean study involving 34 palliative care centres revealed significant variation in pain management outcomes between centres. Higher scores for human resource adequacy were associated with better pain outcomes suggesting that organisational factors played a role in the observed variation in pain outcomes across centres [12].

Despite evidence for between-clinic variations in care practices which may be expected to influence psychosocial outcomes, there has been limited exploration of whether psychosocial outcomes vary across clinics. Our previous study involving radiation oncology clinics indicated that rates of anxiety varied from 24 to $34 \%$; while depression varied from 13 to $20 \%$ [13]. These differences were not statistically significant, and conclusions of the study were limited by the small sample of clinics. To our knowledge, no other studies have explored the question of whether psychological outcomes vary across cancer clinics.

Therefore, the aim of the present study was to examine in a large sample of medical and haematology clinics: (a) whether there is significant variation in patient anxiety and depression across clinics independent of sociodemographic, disease and treatment factors.

\section{Methods}

Setting and design A cross sectional survey was undertaken involving patients recruited from medical oncology and haematology clinics in Australia. Ethics approval, in accordance with the Helskini Declaration of the World Medical Association, was obtained from the Cancer Institute of New South Wales (2011/10/351) and the University of Newcastle Human Research Ethics (H-2010-1324) Committees, as well from ethics committees associated with each participating site.

Recruitment of clinics Medical oncology clinics were eligible if they treated at least 400 new patients each year. A leading medical oncologist from each state acted as a 'clinical champion' to help with recruitment for the study. These individuals identified clinics which met the inclusion criteria, and sent a written invitation. Additional information was provided by phone, email and/or in-person by the research team.

Patient sample Patients with a confirmed diagnosis of cancer, aged 18 years or older, English speaking and presenting for an outpatient haematology or medical oncology consultation were eligible to participate. Those unable to complete the survey independently were excluded. As it would be unreasonable to attribute psychological outcomes to clinic characteristics for patients attending the clinic for the first time, these patients were also excluded.

Recruitment of patients Consecutive patients were recruited in each of the participating clinics. Eligible patients were invited to participate and written informed consent was obtained. The age and sex of non-consenters were collected to assess potential consent bias; no other information was collected due to privacy restrictions. Participants were asked to complete a pen and paper survey either in clinic or at home. Those who took the survey home were asked to return it within 1 week. Non-responders were mailed a second copy of the survey and a reminder letter at 2-3 weeks. A second reminder letter was sent $2-3$ weeks after the first reminder.

\section{Measures}

\section{Explanatory variables}

Demographic variables: Age, sex, highest level of education, Aboriginal and/ or Torres Strait Islander status, marital status, country of birth, home postal code, living situation, employment, private health insurance, concession card status, and 
smoking status were obtained by patient self-report. Concession cards are government issued cards that enable the bearer to obtain discounts on health services and medicines (e.g. for people on low incomes).

Disease and treatment variables included self-reported cancer type, perceived stage of disease at diagnosis (early or advanced), remission status, time since diagnosis, cancer treatments, relocation for treatment and main reason for hospital visit on the day of recruitment.

\section{Outcome variable}

The Hospital Anxiety and Depression Scale (HADS) was used to assess psychological morbidity. The scale includes an anxiety subscale (7 items), and a depression subscale (7 items) [14]. Each item is scored from 0 to 3, giving a maximum score of 21 for each subscale. The HADs has been widely used with cancer patients [15], and meets psychometric criteria for internal consistency, construct validity, and discriminant validity [15]. A score of 8 or above on the depression or anxiety subscales was used to indicate possible caseness for depression or anxiety, respectively [15].

\section{Statistical analysis}

Between-clinic variation in anxiety or depression scores (as determined by the HADS) was initially examined through boxplots, and then formally assessed through random effect linear regression models with anxiety or depression score as the outcome and a random intercept for clinic effect. Modelbased estimates of the site level means (i.e. partially pooled estimates) with 95\% confidence intervals were displayed as caterpillar plots for comparison. The statistical significance of the clinic random effect variance was assessed by comparing the model with a random effect to the equivalent linear model without the random effect, the corresponding likelihood ratio test (LRT) is assumed to follow a 50:50 mixture of chi-square distributions. The intra-class correlation (ICC) was reported, and where clinic variation in anxiety or depression was found to be statistically significant $(p<0.05)$, we reported the corresponding 95\% confidence interval for the ICC obtained from bootstrapping. We also determined if this variation could be explained through differing patient level characteristics by including the following patient demographic (age, sex, marital status, highest level of education, smoking status, concession card status, private health insurance status, Aboriginal and /or Torres Strait Islander status and country of birth) and disease and treatment characteristics (cancer type, time since diagnosis, stage at diagnosis and treatments received) as fixed effects in the mixed-effects regression model and assessed the significance of the between-clinic variation using the method described above. Classification of categorical variables was as shown in Table 1. We also present results for estimates of the
ICC on the probability scale for depression and anxiety dichotomised at a cut point of 8 or more. Logistic regression within a generalised estimating equation framework assuming a compound symmetric residual correlation matrix was used for these analyses.

\section{Sample size}

A sample of approximately 2800 patients from 22 clinics (an average of 127 participants per clinic) would enable a detection of differences in depression or anxiety scores between any two sites of at least 0.35 standard deviation with $80 \%$ power and a $5 \%$ significance level.

\section{Results}

Twenty-two clinics from 19 hospitals participated in the study. Three hospitals had both a medical oncology and a haematology clinic participate. One clinic was located in a private hospital in a regional area, two clinics were located in regional public hospitals, and the remaining 20 clinics were located in public metropolitan hospitals. Most clinics $(n=21)$ were located in teaching hospitals. Two clinics were located in hospitals with less than 200 beds, 8 in hospitals with 200-500 beds and 12 in hospitals with more than 500 beds.

Of the 6036 patients screened, $4233(70 \%)$ were considered eligible. Of these, 3472 agreed to participate (82\%); 2811 returned a completed survey ( $81 \%$ of consenters) and were included in the analysis. There were no statistically significant differences in terms of the gender of consenters and nonconsenters (consenters 57\% female, non-consenters 52\% female, $p=0.056)$. The median number of participants per clinic was 150 (minimum 17, maximum 195). Sociodemographic characteristics of participants are presented in Table 1. Most participants $(n=2561,94 \%)$ reported that they had received most of their cancer care from the hospital from which they were recruited.

\section{Depression scores across treatment clinics}

Mean depression scores ranged from 3.7 to 5.4, with an overall mean of $4.57(\mathrm{SD}=3.78)$. No evidence was found for variation in HADS depression scores among clinics (random effect variance LRT $p$ value $=0.1232$ ), (see Fig. 1 ), therefore no further analysis for the depression outcome was undertaken. The overall proportion of those reporting elevated depression scores $(\geq 8)$ was $21 \%$, $(n=588)$ with rates varying from 12 to $32 \%$ across clinics and an intra-class correlation (ICC) of 0.0001 . 
Table 1 Demographic, disease and treatment characteristics of sample

\begin{tabular}{|c|c|c|c|}
\hline Variable & Category & Oncology $(n=2096) n(\%)$ & Haematology $(n=715) n(\%)$ \\
\hline Gender & Male & $808(39 \%)$ & $408(57 \%)$ \\
\hline Age & Less than 65 & $1187(58 \%)$ & $416(59 \%)$ \\
\hline Aboriginal and Torres Strait Islander origin & Yes & $29(1.4 \%)$ & $7(1.0 \%)$ \\
\hline \multirow[t]{2}{*}{ Marital status } & Married or partnered & $1355(66 \%)$ & $469(66 \%)$ \\
\hline & Single, divorced, separated or widowed & $711(34 \%)$ & $239(34 \%)$ \\
\hline \multirow[t]{2}{*}{ Highest level of education } & High school or below & $1030(50 \%)$ & $321(46 \%)$ \\
\hline & Vocational training, university or other & $1026(50 \%)$ & $384(54 \%)$ \\
\hline \multirow[t]{2}{*}{ Birth place } & Australia & $1441(70 \%)$ & $495(70 \%)$ \\
\hline & Other & $627(30 \%)$ & $214(30 \%)$ \\
\hline Health insured & Yes & $861(42 \%)$ & $374(52 \%)$ \\
\hline Concession card & Yes & $1190(58 \%)$ & $399(56 \%)$ \\
\hline \multirow[t]{3}{*}{ Smoking status } & Current smoker & $206(10 \%)$ & $59(8.3 \%)$ \\
\hline & Former smoker & $882(43 \%)$ & $265(37 \%)$ \\
\hline & Never smoked & $975(47 \%)$ & $389(55 \%)$ \\
\hline \multirow[t]{2}{*}{ Location of residence } & City & $1229(59 \%)$ & $665(94 \%)$ \\
\hline & Regional or remote & $844(41 \%)$ & $39(5.5 \%)$ \\
\hline Living arrangements & With others & $1647(80 \%)$ & $564(80 \%)$ \\
\hline \multirow[t]{3}{*}{ Employment } & Paid employment & $641(31 \%)$ & $244(34 \%)$ \\
\hline & Retired & $847(41 \%)$ & $266(37 \%)$ \\
\hline & $\begin{array}{l}\text { No paid employment (home duties, } \\
\text { unemployed or disability pension) }\end{array}$ & $570(28 \%)$ & $200(28 \%)$ \\
\hline \multirow[t]{8}{*}{ Cancer type } & Breast & $727(36 \%)$ & \\
\hline & Colorectal & $332(16 \%)$ & \\
\hline & Lung & $171(8.4 \%)$ & \\
\hline & Melanoma & $59(2.9 \%)$ & \\
\hline & Other cancer or more than 1 & $564(28 \%)$ & $357(50 \%)$ \\
\hline & Myeloma & $26(1.3 \%)$ & $148(21 \%)$ \\
\hline & Non-Hodgkin lymphoma & $46(2.3 \%)$ & $203(29 \%)$ \\
\hline & Prostate & $117(5.7 \%)$ & \\
\hline \multirow[t]{3}{*}{ Time since diagnosis } & 12 months or less & $992(48 \%)$ & $203(29 \%)$ \\
\hline & 13 to 24 months & $354(17 \%)$ & $121(17 \%)$ \\
\hline & Over 24 months & $723(35 \%)$ & $383(54 \%)$ \\
\hline \multirow[t]{8}{*}{ Treatments ever received } & Surgery & $1475(71 \%)$ & $146(21 \%)$ \\
\hline & Chemotherapy & $1684(82 \%)$ & $575(82 \%)$ \\
\hline & Radiotherapy & $1003(51 \%)$ & $163(23 \%)$ \\
\hline & Hormone therapy & $489(25 \%)$ & $16(2.2 \%)$ \\
\hline & Biological therapy & $250(13 \%)$ & $49(6.9 \%)$ \\
\hline & Bone marrow transplant & $6(0.3 \%)$ & $100(14 \%)$ \\
\hline & Stem cell & $17(0.9 \%)$ & $153(21 \%)$ \\
\hline & Other* & $30(1.5 \%)$ & $55(7.7 \%)$ \\
\hline \multirow[t]{3}{*}{ Cancer stage } & Early & $1113(62 \%)$ & $208(30 \%)$ \\
\hline & Advanced & $507(28 \%)$ & $166(24 \%)$ \\
\hline & Do not know or in remission, or not applicable & $163(9.1 \%)$ & $322(46 \%)$ \\
\hline
\end{tabular}

*Numbers reported for each variable may not add to total sample size due to missing data

\section{Anxiety scores across treatment clinics}

The overall mean anxiety score was $5.24(\mathrm{SD}=4.07)$ with mean scores ranging from 3.9 to 6.4. There was evidence for variation in HADS anxiety scores between clinics (random effect variance LRT $p$ value $=0.0002$ ), with the ICC estimated to be 0.011 (95\% CI $0.005,0.024$ ) (see Fig. 2). The overall proportion of those reporting elevated anxiety scores $(\geq 8)$ was 
Fig. 1 Caterpillar plot of the mean depression scores across haematology and medical oncology clinics (estimated from a random effects model). Modelbased means are a weighted average of site-specific sample mean and the grand mean. Estimates from sites with large variability and/or small sample size are pooled toward to the grand mean

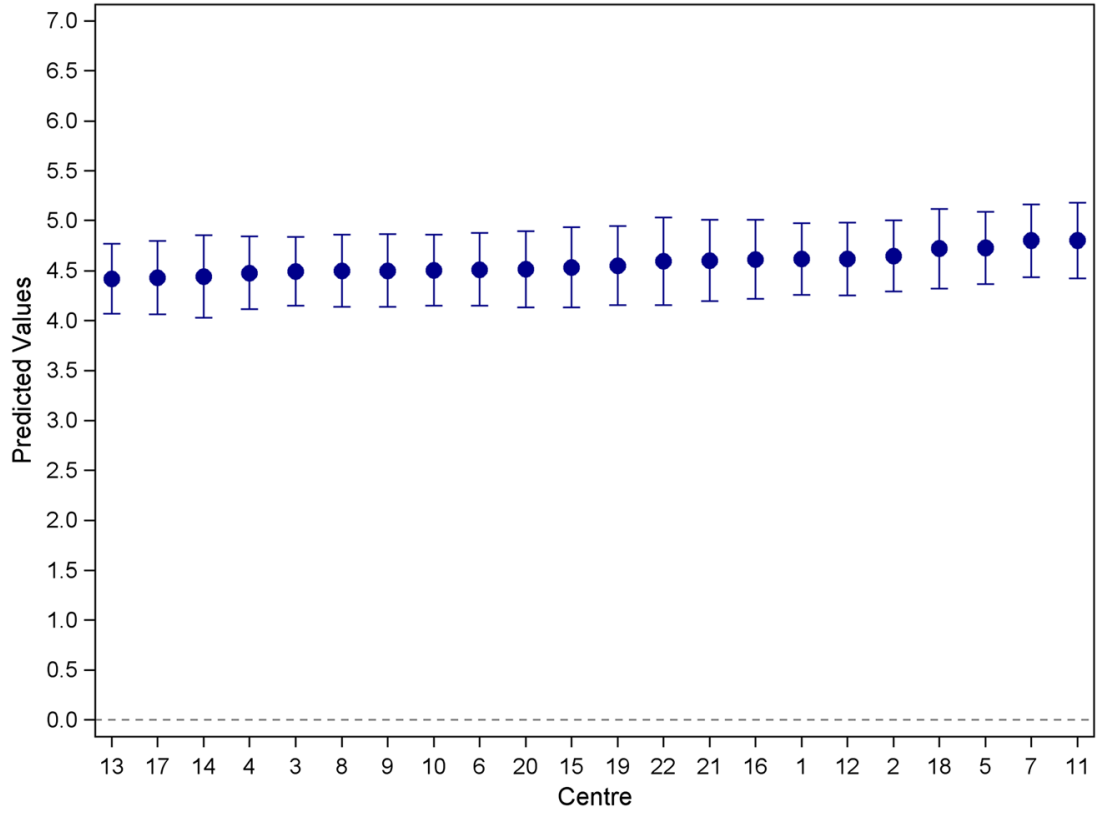

$27 \%$, with rates varying from 12 to $38 \%$ across clinics and an ICC of 0.0067 .

The variation between treatment clinics in HADS anxiety scores was not statistically significantly different than zero once all demographic, disease and treatment predictors were adjusted for (icc $<1 \%$, between-site variation LRT $p=$ $0.1351)$.

\section{Discussion}

The results from this study indicate that approximately one fifth of medical oncology patients reported elevated levels of depression and a quarter indicated clinical levels of anxiety using standardised instruments. These rates are consistent with other studies in the field $[1,2]$ suggesting the external validity of the findings.

\section{No variation in levels of clinical anxiety or depression across cancer treatment clinics}

To our knowledge, this is the first large scale study to examine whether treatment clinics potentially contribute to variation in psychological outcomes for cancer patients. Previous studies suggest that clinic characteristics are associated with unwarranted variation in important outcomes such as pain [12] and
Fig. 2 Caterpillar plot of the mean anxiety scores across haematology and medical oncology clinics (estimated from a random effects model). Modelbased means are a weighted average of site-specific sample mean and the grand mean. Estimates from sites with large variability and/or small sample size are pooled toward the grand mean

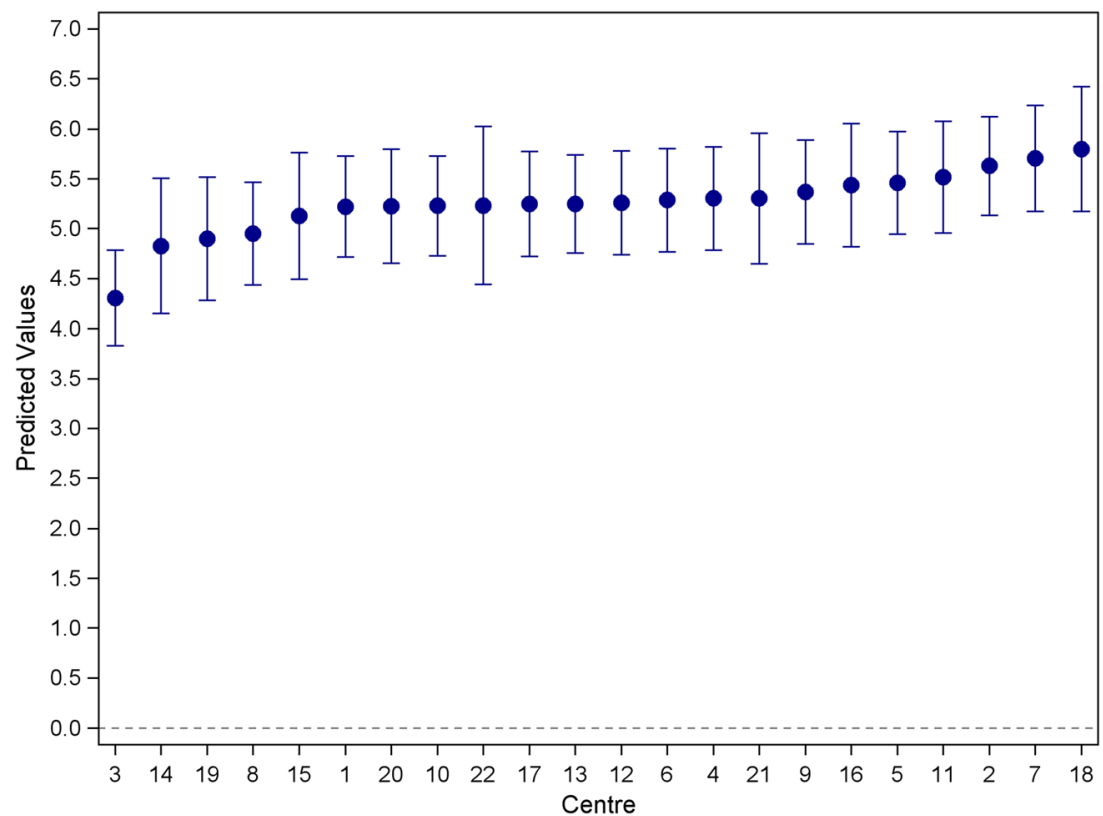


mortality rates following surgery [4]. Jacobsen's study [16] found that indicators of psychosocial care varied across 11 medical oncology clinics; therefore, it is reasonable to expect variation in psychological outcomes may also occur. Therefore, our finding that, once patient factors were controlled for, there was no significant variation across clinics for depression or anxiety is surprising.

A number of hypotheses may explain why between-clinic variation in psychological outcomes was not identified in the present study.

\section{There is a high degree of within-clinic variability in psychosocial care}

In Australia, while clinical practice guidelines were developed for psychosocial care of adults with cancer in 2003 [17], these have subsequently been rescinded. There is no requirement for specific distress screening processes, or provision of specific interventions, in order to gain accreditation. In the absence of policy levers to ensure implementation of specific care processes, individual clinicians have a high level of discretion regarding the processes (or lack thereof) which they put in place to detect and treat anxiety, depression and related outcomes. Therefore, our results may reflect that within clinic variability in the process of care used by clinicians within a clinic was greater than between clinic variation in processes.

\section{There is a high degree of similarity in the psychosocial care provided across clinics}

It is also possible that there is little within-clinic variation, and that clinics implement very similar care processes. This would also account for our finding of no variation in outcomes. While private hospitals represent approximately $47 \%$ of all hospitals in Australia [18], only one private hospital was included in our sample due to prohibitive costs associated with obtaining research ethics and governance approvals. A greater representation of private clinics may have introduced greater variability as it is the nature of private enterprise to strive to differentiate oneself from the crowd by applying innovations in products and processes.

\section{Efficacious psychosocial care strategies are not effective in real world settings, hence variability (or lack of) in delivery across clinics does not impact on outcomes}

While numerous studies have demonstrated the efficacy of specific treatments to improve the psychological well-being of cancer patients [19-22], effectiveness studies are more limited. Efficacy studies are undertaken in optimal circumstances with a focus on internal validity [23]. Often, this is achieved by controlling for context in a way that does not reflect the real world. Patient eligibility criteria, for example, are carefully delineated (often excluding individuals with commonly occurring co-morbidities, culturally diverse backgrounds and limiting age range) [23]. Furthermore, clinicians involved in intervention trials may be biased toward those who are already committed to improving psychological care. Additionally, in well-controlled research, the fidelity of adherence to the treatment protocol is usually carefully monitored and maintained [23]. These experimental conditions may not be achieved in everyday clinical practice, diluting the potential effectiveness of an intervention and limiting the generalisability of results. Therefore, if efficacious interventions are implemented poorly, or are ineffective in real-world settings, then variability in care processes may not translate into variability in psychological outcomes of patients. There is a need for the field to undertake effectiveness studies (sometimes referred to as pragmatic trials) where the power of the intervention is examined in real-world circumstances. In such studies, the evaluation design is able to take proper account of context by allowing a more realistic eligibility criteria, ensuring a range of clinicians are involved, and allowing for the expected variation in treatment fidelity.

\section{Measurement error may have hindered the ability to identify variability across clinics}

The Hospital Anxiety and Depression Scale is a commonly used measure of psychosocial well-being. It has acceptable psychometric properties, and scores are not confounded by the physical symptoms of cancer or its treatment [15]. However, it remains a screening tool, and unlike a structured clinical interview, is not diagnostic. Therefore, it is possible that measurement error may have impacted on the accuracy of the anxiety and depression scores. In an effort to overcome uncertainty about the optimal clinical cut-point for cancer populations [13], analysis for this paper was undertaken using the HADS score as a continuous variable. Examining the HADS score in this manner increases the statistical power of the analysis.

\section{Limitations}

As discussed above, our results must be interpreted in light of several limitations which may have affected the results. These include potential measurement error, and a hospital sample which consisted almost entirely of large metropolitan public hospitals. Further, our study did not allow us to rule out possible explanations for the lack of variability observed such as there being a high degree of within clinic variability in the delivery of care. 


\section{Conclusions}

This study provides the first large scale examination of whether variability in psychological outcomes occurs across oncology clinics. Our results indicate that there is no variation in anxiety and depression outcomes across medical oncology and haematology clinics.

Acknowledgments Our thanks to the cancer patients and participating cancer treatment centres; Rochelle Smits, Alison Zucca, Heidi Turon and Hannah Small for research support; Sandra Dowley for data management; and Alessandra Bisquerra and Tiffany Evans for statistical assistance.

Funding This research was funded by a National Health and Medical Research Council Project Grant (ID 1010536), a Strategic Research Partnership Grant (CSR11-02) from Cancer Council NSW to the Newcastle Cancer Control Collaborative (New-3C), infrastructure funding from the Hunter Medical Research Institute (HMRI) [no grant number applicable], National Health and Medical Research Council (NHMRC) Translating Research into Practice Fellowship (ID 1073031) to MC, NHMRC Career Development Fellowship (ID 1061335) to CP, NHMRC (ID 1073317) and Cancer Institute New South Wales (13/ECF/ 1-37) Early Career Fellowships to AB, and Leukaemia Foundation of Queensland Post-doctoral Fellowship to TCM.

\section{Compliance with ethical standards}

Conflicts of interest All authors declare that they have no conflicts of interest.

Ethical approval All procedures performed in studies involving human participants were in accordance with the ethical standards of the institutional and/or national research committee and with the 1964 Helsinki declaration and its later amendments or comparable ethical standards.

Informed consent Informed consent was obtained from all individual participants included in the study.

Open Access This article is distributed under the terms of the Creative Commons Attribution-NonCommercial 4.0 International License (http:// creativecommons.org/licenses/by-nc/4.0/), which permits any noncommercial use, distribution, and reproduction in any medium, provided you give appropriate credit to the original author(s) and the source, provide a link to the Creative Commons license, and indicate if changes were made.

\section{References}

1. Boyes AW, Girgis A, D'Este CA, Zucca AC, Lecathelinais C (2013) Prevalence and predictors of the short-term trajectory of anxiety and depression in the first year after a cancer diagnosis: a population-based longitudinal study. J Clin Oncol 31(21):2724 2732. https://doi.org/10.1200/JCO.2012.44.7540

2. Linden W, Vodermaier A, MacKenzie R, Greig D (2012) Anxiety and depression after cancer diagnosis: prevalence rates by cancer type, gender, and age. J Affect Disord 141(2):343-351

3. Mitchell AJ, Chan M, Bhatti H, Halton M, Grassi L, Johansen C, Meader N (2011) Prevalence of depression, anxiety, and adjustment disorder in oncological, haematological, and palliative-care settings: a meta-analysis of 94 interview-based studies. Lancet Oncol 12(2):160-170. https://doi.org/10.1016/S1470-2045(11)70002-X

4. Birkmeyer JD, Stukel TA, Siewers AE, Goodney PP, Wennberg DE, Lucas FL (2003) Surgeon volume and operative mortality in the United States. N Engl J Med 349(22):2117-2127. https://doi. org/10.1056/NEJMsa035205

5. Forsythe LP, Kent EE, Weaver KE, Buchanan N, Hawkins NA, Rodriguez JL, Blythe Ryerson A, Rowland JH (2013) Receipt of psychosocial care among cancer survivors in the United States. J Clin Oncol 31(13):1961-1969

6. Carver C, Pozo C, Harris S, Noriega V, Scheier M, Robinson D, Ketcham A, Moffat F, Clark K (1993) How coping mediates the effect of optimism on distress: a study of women with early stage breast cancer. J Pers Soc Psychol 62(5):375-390

7. Applebaum AJ, Stein EM, Lord-Bessen J, Pessin H, Rosenfeld B, Breitbart W (2014) Optimism, social support, and mental health outcomes in patients with advanced cancer. Psychooncology 23: 299-306. https://doi.org/10.1002/pon.3418

8. Carlson LE, Angen M, Cullum J, Goodey E, Koomans J, Lamont L, MacRae JH, Martin M, Pelletier G, Robinson J, Simpson JSA, Speca M, Tillotson L, Bultz BD (2004) High levels of untreated distress and fatigue in cancer patients. Br J Cancer 90:2297-2304. https://doi.org/10.1038/sj.bjc.6601887

9. Mcillmurray MB, Thomas C, Francis B, Morris S, Soothill K, AlHamad A (2001) The psychosocial needs of cancer patients: findings from an observational study. Eur J Cancer Care 10(4):261269. https://doi.org/10.1046/j.1365-2354.2001.00280.x

10. Moyer A (1997) Psychosocial outcomes of breast-conserving surgery versus mastectomy: a meta-analytic review. Health Psychol 16(3):284-298. https://doi.org/10.1037/0278-6133.16.3.284

11. Goodney P, Dzebisashvili D, DC G, KK B (2015) Variation in the care of surgical conditions. Dartmouth Atlas of healthcare series. The Dartmouth Institute of Health Policy and Clinical Practice, Hanover. https://doi.org/10.1024/0301-1526/a000463

12. Shin DW, Hwang SS, Oh J, Kim JH, Park JH, Cho J, Cho B, Jung KT, Park E (2012) Variations in pain management outcomes among palliative care centers and the impact of organizational factors. Cancer 118:5688-5697. https://doi.org/10.1002/cncr.26722

13. Carey M, Paul C, Mackenzie L, Sanson-Fisher R, Cameron E (2012) Do cancer patients' psychosocial outcomes and perceptions of quality of care vary across radiation oncology treatment centres? Eur J Cancer Care 21(3):384-389. https://doi.org/10.1111/j.13652354.2011.01299.x

14. Zigmond A, Snatih R (1983) The Hospital anxiety and depression scale. Acta Psychiatr Scand 67:361-370. https://doi.org/10.1111/j. 1600-0447.1983.tb09716.x

15. Luckett T, Butow PN, King MT, Oguchi M, Heading G, Hackl NA, Rankin N, Price MA (2010) A review and recommendations for optimal outcome measures of anxiety, depression and general distress in studies evaluating psychosocial interventions for Englishspeaking adults with heterogeneous cancer diagnoses. Support Care Cancer 18(10):1241-1262. https://doi.org/10.1007/s00520-0100932-8

16. Jacobsen PB, Shibata D, Siegel M, Lee JH, Fulp WJ, Alemany C, Abesada-Terk G, Brown R, Cartwright T, Faig D, Kim G, Levine R, Markham MJ, Schreiber F, Sharp P, Malafa M, Shibata D, Siegel M, Lee JH, Fulp WJ, Alemany C, Abesada-Terk G, Brown R, Cartwright T, Faig D, Kim G, Levine R, Markham MJ, Schreiber F, Sharp P, Malafa M (2010) Evaluating the quality of psychosocial care in outpatient medical oncology settings using performance indicators. Psychooncology

17. National Breast Cancer Centre, National Cancer Control Initiative (2003) Clinical practice guidelines for the psychosocial care of adults with cancer. National Breast Cancer Centre, Camperdown 
18. Australian Institute of Health and Welfare (2016) Australia's hospitals $2014-15$ at a glance. AIHW, Canberra

19. Jassim GA, Whitford DL, Hickey A, Carter B (2015) Psychological interventions for women with non-metastatic breast cancer. Cochrane Database Syst Rev 5. https://doi.org/10.1002/14651858. CD008729.pub2

20. Parahoo K, McDonough S, McCaughan E, Noyes J, Semple C, Halstead E, Neuberger M, Dahm P (2013) Psychosocial interventions for men with prostate cancer. [Review] Cochrane Database Syst Rev

21. Semple C, Parahoo K, Norman A, McCaughan E, Humphris G, Mills M (2013) Psychosocial interventions for patients with head and neck cancer. [Review] Cochrane Database Syst Rev. https://doi. org/10.1002/14651858.CD009441.pub2

22. Zhang M, Wen Y, Liu W, Peng L, Wu X, Liu Q (2015) Effectiveness of mindfulness-based therapy for reducing anxiety and depression in patients with cancer: a meta-analysis. Medicine 94(45): e0897-e0890. https://doi.org/10.1097/MD. 0000000000000897

23. Green L, Glasgow R (2006) Evaluating the relevance, generalization and applicability of research. Issues in external validation and translation methodology. Eval Health Prof 29(1):126-153 